\title{
New cell line development for antibody- producing Chinese hamster ovary cells using split green fluorescent protein
}

Yeon-Gu Kim, Byoungwoo Park, Jung Oh Ahn, Joon-Ki Jung, Hong Weon Lee and Eun Gyo Lee*

\begin{abstract}
Background: The establishment of high producer is an important issue in Chinese hamster ovary (CHO) cell culture considering increased heterogeneity by the random integration of a transfected foreign gene and the altered position of the integrated gene. Fluorescence-activated cell sorting (FACS)-based cell line development is an efficient strategy for the selection of $\mathrm{CHO}$ cells in high therapeutic protein production.

Results: An internal ribosome entry site (IRES) was introduced for using two green fluorescence protein (GFP) fragments as a reporter to both antibody chains, the heavy chain and the light chain. The cells co-transfected with two GFP fragments showed the emission of green fluorescence by the reconstitution of split GFP. The FACS-sorted pool with GFP expression had a higher specific antibody productivity $\left(q_{\mathrm{Ab}}\right)$ than that of the unsorted pool. The $q_{\mathrm{Ab}}$ was highly correlated with the fluorescence intensity with a high correlation coefficient, evidenced from the analysis of median GFP and $q_{\mathrm{Ab}}$ in individual selected clones.

Conclusions: This study proved that the fragment complementation for split GFP could be an efficient indication for antibody production on the basis of high correlation of $q_{A b}$ with reconstitution of GFP. Taken together, we developed an efficient FACS-based screening method for high antibody-producing $\mathrm{CHO}$ cells with the benefits of the split GFP system.
\end{abstract}

Keywords: Split GFP, FACS, Cell line development, Antibody production, CHO cells

\section{Background}

Chinese hamster ovary $(\mathrm{CHO})$ cells are one of the most widely used host cells for therapeutic protein production. The selection of $\mathrm{CHO}$ cells for high therapeutic protein production is of particular interest in $\mathrm{CHO}$ cell culture for a wide variety of individual clones for productivity from a random integration and gene amplification system [1]. Due to the large number of candidates that need their productivity evaluated, an efficient highthroughput cell screening system needs to be developed.

Flow cytometry can efficiently evaluate a number of cells at the single-cell level in a short time and isolate a single clone from sub-populations. Various fluorescenceactivated cell sorting (FACS)-based cell screening methods have been developed for $\mathrm{CHO}$ cells with the benefits of flow cytometry [1]. The use of an antibody conjugated

\footnotetext{
* Correspondence: eglee@kribb.re.kr

Process Engineering Center, KRIBB, Daejeon 305-806, Korea
}

with a fluorescent molecule, which is the target to the membrane-retained therapeutic proteins has been used to find high-producing clones in the combination with FACS. Although its mechanism has not been fully elucidated, the incubation of antibody-producing $\mathrm{CHO}$ cells at $4^{\circ} \mathrm{C}$ could lead to the transient association of the desired antibody with the cell surface [2]. The target antibody at cell surface can be detected by another fluorescentconjugated antibody and the highly fluorescent-labeled clones are isolated as high producers using FACS system, evidenced from correlating the mean fluorescence intensity with specific productivity $(q)$. Similarly, to capture the secreted protein in a small droplet or matrix generated on the cell surface, gel microdrop technology and affinity capture surface display method combined with fluorescentconjugated antibody have been developed [3,4]. Instead of direct measurement of desired antibody by anchoring membrane, the cell surface reporter, CD20 not normally expressed in $\mathrm{CHO}$ cells, is co-expressed with the target

\section{() Biomed Central}


protein by internal ribosome entry site (IRES)-linking and the high producer is detected using a fluorescent-conjugated anti-CD20 antibody [5].

Being different from the direct detection of a target protein, intracellular fluorescent materials, such as green fluorescence protein (GFP) and yellow fluorescence protein (YFP), were a widely used fluorescent protein for FACS because of easy activation without co-factors and substrates. Meng et al. (2000) evaluated the usefulness of GFP as an indicator for finding high-producing clone when it is co-expressed with therapeutic protein in one vector system [6]. Due to the close relationship between fluorescence intensity and $q$, clones with high $q$ could easily be isolated from sub-populations based on fluorescence level using FACS. In a similar way, clones with high fluorescence level were selected as for high $q$ in FITC-conjugated MTX-mediated gene amplification system on the basis of correlation of $q$ with amplified gene copy number [7]. However, in the case of isolation of antibody-producing cells, there is a limitation in the applications of these systems because antibody is composed of two fragment proteins which are heavy and light chains. Therefore, Sleiman et al. (2008) developed two color fluorescent protein-based FACS to select clone possessing the high level of the heavy chain and the light chain by detecting GFP and YFP, respectively [8].

For the characteristics of an antibody composed of two fragments, a heavy chain and a light chain, the fragment complementation system can be useful in finding antibody-producing cells. Previously, Bianchi and McGrew (2003) developed an efficient system for the selection of $\mathrm{CHO}$ cells with high levels of both antibody chains using a DHFR fragment [9]. The fragment complementation systems for split GFP by means of anti-parallel leucine zipper and EF-hand calcium binding motifs of calbindin D9k have been reported [10-12]. Expression of two fragments of GFP does not achieve folding and fluorescence of GFP by itself. However, GFP is reassembled through the introduction of strong mediator such as anti-parallel leucine zipper and EF-hand calcium binding motifs of calbindin D $9 k$ by non-covalent reconnection. Despite the merits in using split GFP, such as the extensive use of flow cytometry and efficient complementation system for GFP, there is, to date, no report introducing it related to cell line development in mammalian cell culture. In the present study, we developed a new cell screening method for high antibody-producing $\mathrm{CHO}$ cells based on the reassembly of split GFP combined with FACS.

\section{Results and discussion}

To evaluate the role of split GFP as a reporter for antibody production, we constructed an overexpression vector for split GFP and antibody chains expressed simultaneously. Figure $1 \mathrm{~A}$ shows the schematic diagram representing the way the assembly of split GFP works as a reporter for the selection of antibody-producing cells. The GFP fragments, called N-GFP and C-GFP (described in the 'Methods' section), are co-linked with a light chain and heavy chain gene using an IRES sequence, respectively. These constructs containing an IRES sequence, pNGFP-Light and pCGFPHeavy, led us to hypothesis that the transcription level of the light and heavy chains is highly correlated with that of N-GFP and C-GFP. From this hypothesis, our speculation extends to the idea that the high GFP-expressing clone may be the high antibody-producing clone.

To confirm both the antibody expression and the assembly of the GFP with a constructed vector, a bicistronic vector containing GFP fragments and an antibody gene, pNGFP-Light and pCGFP-Heavy, was transfected into HEK293T cells. As a control, a GFP-overexpressing vector, pEGFP-C1, was also transfected into HEK293T cells. Figure 1B shows the confocal microscopic images of transfected cells and antibody titer produced from them. The emission of green fluorescence from reconstituted split GFP was efficiently detected when the two fragments were co-expressed. In addition, the cells with green fluorescence could produce the antibody simultaneously. The same results were confirmed in CHO-K1 cells (data not shown). Accordingly, we proceeded with these constructs to evaluate the efficiency of this screening system in $\mathrm{CHO}$ cells.

To investigate whether the sorted cells by FACS are the antibody-producing $\mathrm{CHO}$ cells, the $\mathrm{CHO}$ cells with intracellular green fluorescence (First sort pool and Second sort pool) were generated by two rounds of FACS from the CHO cells co-transfected with pNGFP-Light and pCGFP-Heavy (Unsorted pool). Figure 2 shows the flow cytometry analysis and specific antibody productivity $\left(q_{\mathrm{Ab}}\right)$ of unsorted and FACS-sorted cells. The discrimination of cells with green fluorescence was performed on a FITC/SSC diagram. The percentage of the cell population with GFP expression in the First sort pool and Second sort pool was much higher than that in the Unsorted pool. Interestingly, the increase in $q_{\mathrm{Ab}}$ was highly associated with the increase in the cell population with GFP expression. Taken together, we found that the FACS-sorted cells with GFP expression are the antibody-producing $\mathrm{CHO}$ cells.

To evaluate the efficiency of split GFP-based clone selection for high antibody-producing cells, individual clones were isolated from the Unsorted pool and the Second sort pool by the limiting dilution method. Table 1 shows the distribution of 116 isolated clones from the Unsorted pool and the Second sort pool with regard to antibody titer, respectively. All of the Unsorted poolderived clones have low antibody production (less than $1 \mathrm{mg} / \mathrm{L})$. Eighty-two out of 116 clones in the Second sorted pool have antibody production exceeding $1 \mathrm{mg} / \mathrm{L}$. Moreover, there are four clones having over $10 \mathrm{mg} / \mathrm{L}$ in 


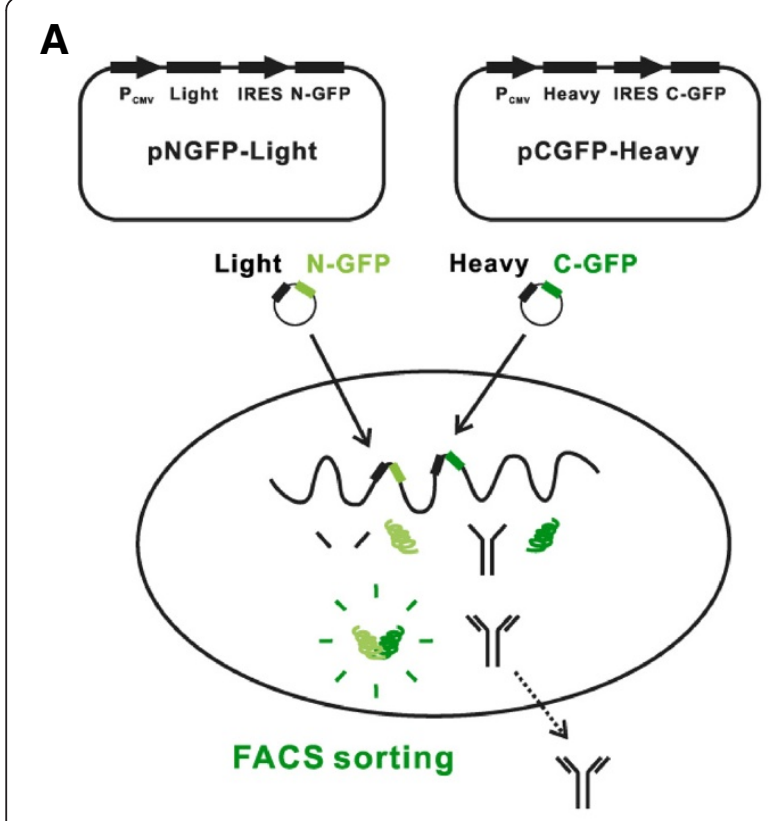

ELISA

B

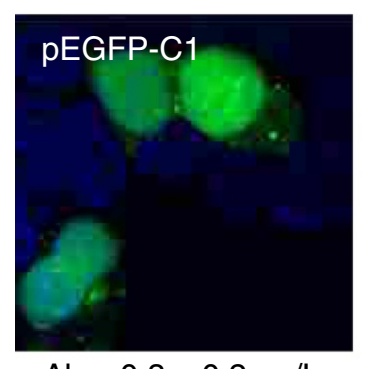

$\mathrm{Ab}=0.3 \quad 0.2 \mu \mathrm{g} / \mathrm{L}$

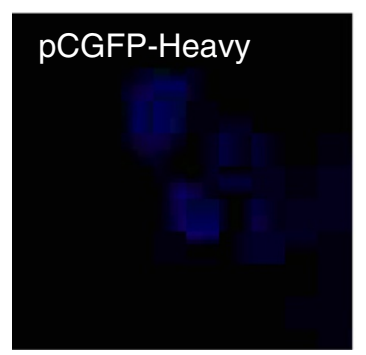

$A b=0.1 \quad 0.0 \mu \mathrm{g} / \mathrm{L}$

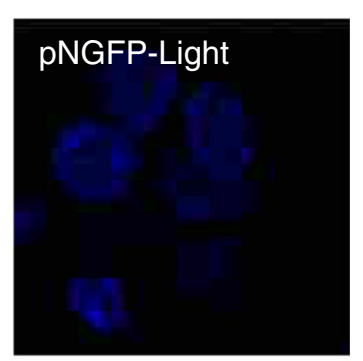

$\mathrm{Ab}=0.2 \quad 0.1 \mu \mathrm{g} / \mathrm{L}$

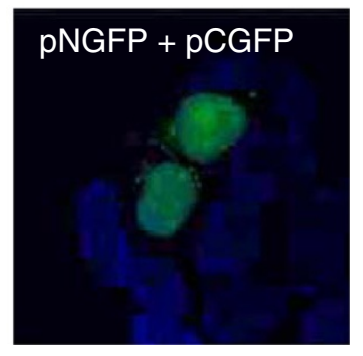

$\mathrm{Ab}=35.0 \quad 3.6 \mu \mathrm{g} / \mathrm{L}$

Figure $1 \mathrm{New}$ cell line development for high antibodyproducing mammalian cells. (A) Schematic diagram for the split GFP-based cell screening method. (B) Confocal microscopic images of cells transfected with a GFP-overexpressing vector (pEGFP-C1) or bicistronic vector having GFP fragements and both antibody chains (pNGFP-Light and pCGFP-Heavy). After transfection with these vectors, the cells were cultivated for two days at $30^{\circ} \mathrm{C}$. DAPI was used for nucleus staining. Antibody titer was quantified from culture supernatant by ELISA.

the Second sort pool-derived clones, while there are none in the Unsorted pool-derived clones. Altogether, the clone selection with split GFP-based FACS appears to be an efficient strategy to select high antibodyproducing $\mathrm{CHO}$ cells.

A major issue in the development of a cell screening method using a reporter is to verify the co-relationship between the expression level of the reporter and the antibody. Especially, the screening system dealing with intracellular reporter should be evaluated because antibody is generally secreted protein. In the same context, previous reports utilizing a fluorescent protein as an intracellular reporter proved the mutual relation between productivity of desired protein and fluorescence intensity $[6,8]$.

To address this issue, the median GFP and $q_{\mathrm{Ab}}$ of 30 selected clones were estimated. Figure 3 shows the relationship of the median GFP and $q_{\mathrm{Ab}}$ for individual selected clones. The $q_{\mathrm{Ab}}$ is highly correlated with the fluorescence intensity as indicated by the high correlation coefficient $\left(R^{2}=0.8947\right)$. Accordingly, we confirmed that the selected clone having high fluorescence intensity by GFP-based cell sorting is the high antibody producer with high $q_{\mathrm{Ab}}$.

Another issue that arises with the usage of intracellular reporter is the determination of the optimum time-point for sorting and metabolic burden by co-expressing reporter gene. Unlike the use of non-split fluorescent protein, we can exclude the issue of determining the optimal point in split fluorescent protein because the split fluorescent protein can be assembled at specific conditions, such as low temperature in the case of split GFP. Previously, Bochkov and Palmenberg (2006) showed that the level of gene expression linked with attenuated IRES was about 11-fold lower than wild-type IRES [13]. Also, the modified form of GFP with increased fluorescence intensity was reported [14]. The introduction of attenuated IRES and improved form of GFP can be a solution to reduce the metabolic burden of fluorescent protein.

The intracellular fluorescent protein-based FACS for clone selection in $\mathrm{rCHO}$ cells is a reasonable strategy as it does not require complex preparation steps to capture and detect a secreted antibody. Previously, Sleiman et al. (2008) developed an efficient dual intracellular autofluorescent protein-associated FACS to select antibodyproducing $\mathrm{CHO}$ cells with a high level of both antibody chains [8]. In this system, two reporters, GFP and YFP, are applied for the heavy and light chain, respectively. In general, a correct compensation is necessary for accurate analysis when more than two colors are used as a parameter in flow cytometry [15]. Moreover, a similar level of fluorescence intensity in GFP and YFP does not guarantee a similar expression level of the heavy chain and the light 


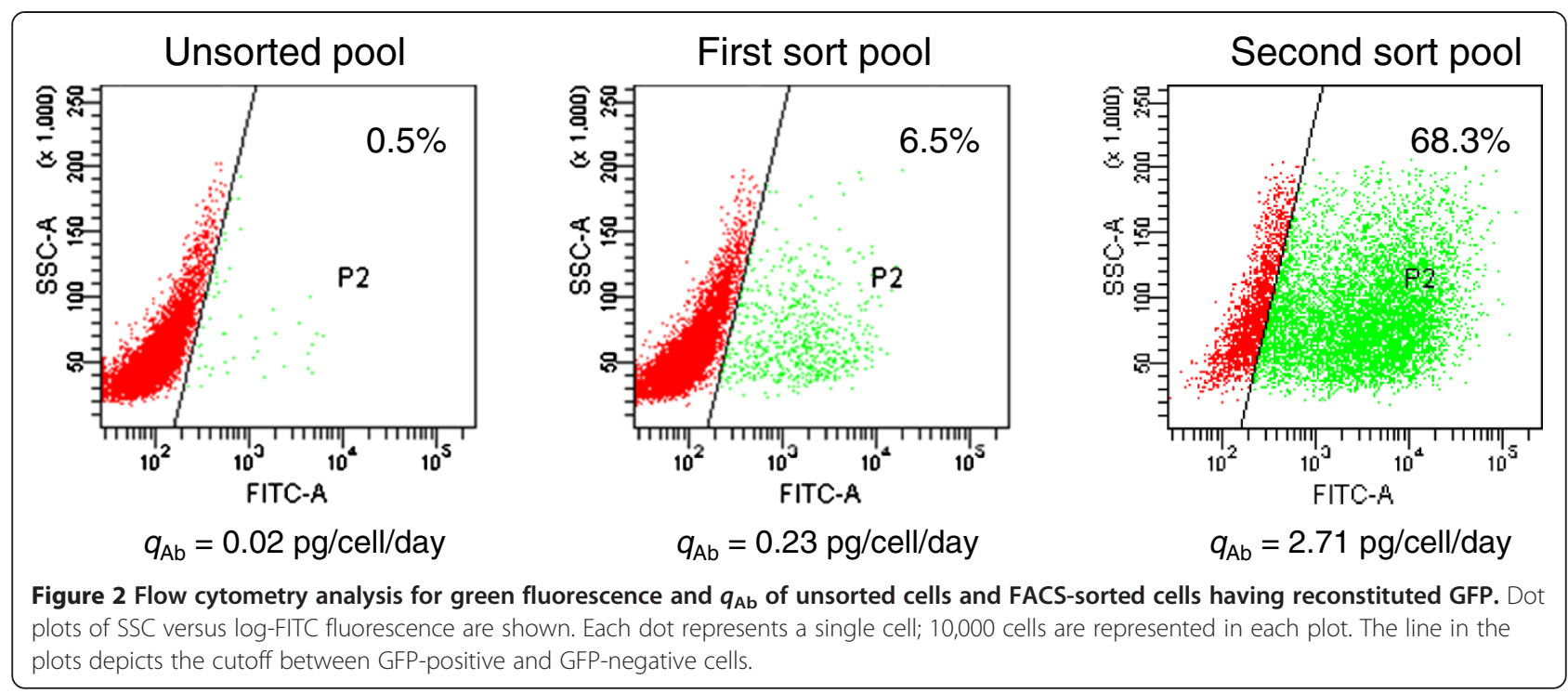

chain because fluorescence intensity is a relative value, not an absolute value. Also, the split GFP-based cell screening method developed in this study is advantageous in that one reporter is introduced for quantitative analysis. By employing this type of complementation system, we can omit the process of compensation.

The additional validation for dynamic range of the GFP assay and long-term stability of constructed cell line may be needed to successfully apply the split GFPbased cell screening method for commercial production cell line. The expanded result for dynamic range of GFP expression in high antibody-producing clones with enhanced $q_{\mathrm{Ab}}$ via gene amplification system and/or cisacting element for augmenting gene expression may be helpful to meet the industrial standard. The genetic instability of antibody-producing $\mathrm{CHO}$ cell line during long-term culture in the absence of selective pressure is one of main issues in $\mathrm{CHO}$ cell culture [16]. The data provided from long-term culture with constructed cell lines based on split GFP system to determine the association of GFP expression with reduced $q_{\mathrm{Ab}}$ will be an interesting extension of this work.

Table 1 Distribution of isolated clones from the Unsorted pool and the Second sort pool for antibody titer

\begin{tabular}{lll}
\hline Criterion $(\mathbf{m g} / \mathbf{L})^{\mathbf{a}}$ & Unsorted pool & Second sort pool \\
\hline$<0.5$ & $115 / 116$ & $33 / 116$ \\
$0.5-1$ & $1 / 116$ & $1 / 116$ \\
$1-5$ & $0 / 116$ & $28 / 116$ \\
$5-10$ & $0 / 116$ & $50 / 116$ \\
$>10$ & $0 / 116$ & $4 / 116$
\end{tabular}

${ }^{a}$ Cells were inoculated at a concentration of $3 \times 10^{5}$ cells $/ \mathrm{mL}$ into a 6-well plate. The samples were measured by ELSIA after 3-days cultivation.

\section{Conclusions}

The considerable clonal variation attributed from transfection and gene amplification makes it difficult to establish high-producing $\mathrm{rCHO}$ cell line. For large number of analytes, it is necessary to develop an efficient highthroughput cell screening system. In this study, we developed an efficient screening method based on reconstitution of split GFP to select high antibody-producing $\mathrm{CHO}$ cells using a FACS analysis. On the basis of correlation between $q$ and fluorescence intensity by reconstituting GFP, the fragment complementation system for split GFP could be a powerful tool for antibody production in $\mathrm{CHO}$ cells.

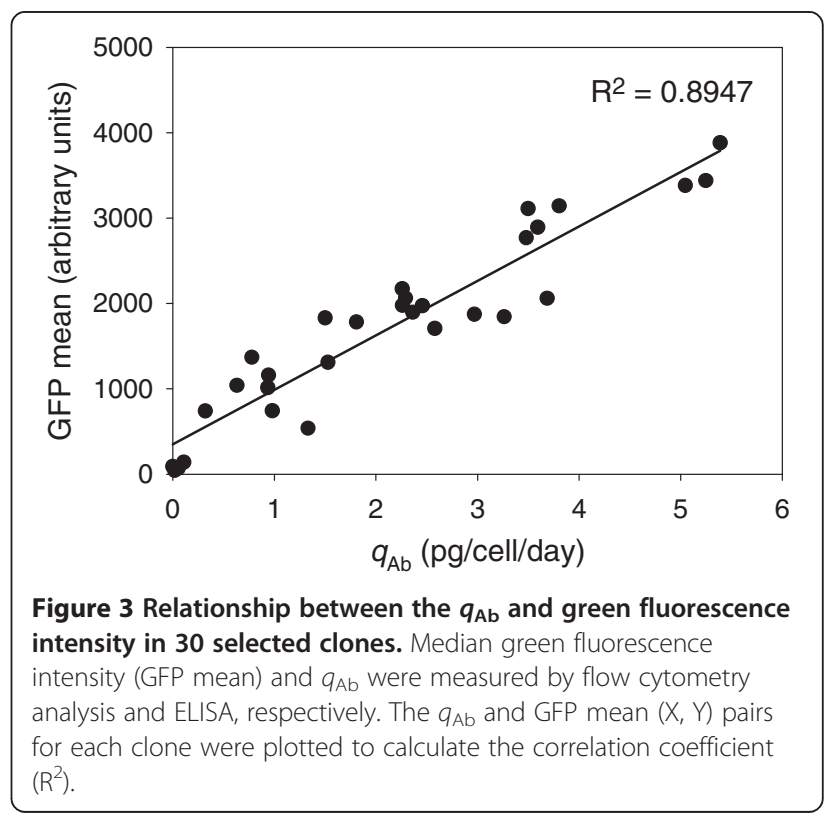




\section{Methods}

\section{Plasmid construction}

A modified pIRES vector (BD Biosciences Clontech) with wild-type IRES was used for the construction of pNGFPLight and pCGFP-Heavy. The light and heavy chain genes were kindly provided by Dr. J. S. Yoo, Pharmabcine Co., Ltd. They were inserted into MCS-A of the pIRES vector to yield pIRES-Light and pIRES-Heavy, respectively. The GFP coding sequence was obtained from pIRES2-EGFP (BD Biosciences Clontech) by PCR amplification. DNA construct for Calbindin D9k including EF1 and EF2 were synthesized in Bioneer Co., Ltd. as described previously [10]. N-GFP encoding N-terminal GFP (amino acid residue 1-158) linked with EF1 via GGSGSGSS and C-GFP encoding C-terminal GFP (amino acid residue 159-239) linked with EF2 via TSGGSG were made by a sequential PCR method as described previously [12,17]. Then, they were inserted into MCS-B of the pIRES vector resulting in pNGFPLight and pCGFP-Heavy, respectively.

\section{Cell line and culture maintenance}

The CHO-K1 cells (ATCC CRL-61) were plated onto $25 \mathrm{~cm}^{2}$ T-flasks (Nunc), and attached for $24 \mathrm{~h}$ before transfection. The $\mathrm{CHO}-\mathrm{K} 1$ cells $\left(1 \times 10^{6}\right.$ cells $)$ were cotransfected with pNGFP-Light $(4 \mu \mathrm{g})$ and/or pCGFPHeavy $(4 \mu \mathrm{g})$ using Lipofectamine ${ }^{\mathrm{TM}} 2000$ (Invitrogen) according to the manufacturer's protocol. Drug selection was carried out for three weeks by seeding $1 \times 10^{4}$ cells/ $\mathrm{mL}$ in $75 \mathrm{~cm}^{2}$ T-flasks (Nunc) containing Roswell Park Memorial Institute (RPMI; Invitrogen) supplemented with $10 \%$ fetal bovine serum (FBS; Invitrogen) and $500 \mu \mathrm{g} / \mathrm{mL}$ G418 (Invitrogen). After drug selection, a stable pool, called the Unsorted pool, was maintained for three days at a concentration of $1 \times 10^{5}$ cells $/ \mathrm{mL}$. The Unsorted pool was cultivated for two days at $30^{\circ} \mathrm{C}$ and then the top $1 \%$ of the fluorescence intensity in the Unsorted pool using FACS analysis (See below) was collected and called the First sort pool. The Second sort pool was prepared from the top $1 \%$ of the fluorescence intensity of the First sort pool using the same procedure.

\section{Batch culture}

Exponentially growing cells were inoculated at a concentration of $0.7 \times 10^{5}$ cells $/ \mathrm{mL}$ into $25 \mathrm{~cm}^{2}$ T-flasks (Nunc) containing $5 \mathrm{~mL}$ of RPMI supplemented with 10\% FBS and $500 \mu \mathrm{g} / \mathrm{mL} \mathrm{G418}$, and the plates were incubated in a humidified $5 \% \mathrm{CO}_{2}$ /air mixture at $37^{\circ} \mathrm{C}$. Periodically, the $25 \mathrm{~cm}^{2}$ T-flasks were sacrificed for determining the viable cell concentration. The cell concentration and viability were estimated with a Cytolecon automated cell imaging counter (CYT-100; ECI Inc.) using the trypan blue dye exclusion method. Culture supernatants, after centrifugation, were aliquoted and kept frozen at $-70^{\circ} \mathrm{C}$ for later analyses.

\section{Enzyme-linked immunosorbent assay (ELISA)}

The secreted antibody concentration was quantified by an enzyme linked immunosorbent assay (ELISA). Briefly, 96-well plates (Nunc) were coated with anti-human IgG (Sigma) and blocked with $2 \%$ bovine serum albumin (BSA; Sigma) in phosphate buffered saline (PBS) containing 0.1\% Tween-20 (Sigma). Human IgG standard (Sigma) and culture supernatants diluted with a blocking buffer were loaded into each well and treated with peroxidase conjugated goat anti-human IgG (Sigma) in the blocking buffer. 3,3',5,5'-tetramethyl benzidine (TMB; Sigma) was used as a substrate and $1 \mathrm{M} \mathrm{H}_{2} \mathrm{SO}_{4}$ was used to stop the reaction. Absorbance was measured by an UV ELISA reader (Bio- $\mathrm{Rad}$ ) at $450 \mathrm{~nm}$.

\section{Fluorescence-activated cell sorting (FACS) analysis}

The cells were cultivated for two days at $30^{\circ} \mathrm{C}$ and then were resuspended with PBS. The FACSAria ${ }^{\mathrm{TM}}$ system (BD Biosciences Clontech) was used to estimate the green fluorescence intensity of various clones as described previously [18]. Green fluorescence at $525 \mathrm{~nm}$ was detected through an FL1 set at a PMT voltage of 400, with a logarithmic gain. Ten thousand cells were analyzed for each sample.

\section{Fluorescence microscopy}

The HEK293T cells (ATCC CRL-11268) were plated onto RS-treated Lab-Tek ${ }^{\circledR}$ II Chamber Slides (Nunc), and attached for $24 \mathrm{~h}$ before transfection. For transient expression, HEK293T cells $\left(1 \times 10^{5}\right.$ cells $)$ were co-transfected with pNGFP-Light $(0.4 \mu \mathrm{g})$ and/or pCGFP-Heavy $(0.4 \mu \mathrm{g})$ using Lipofectamine ${ }^{\mathrm{TM}} 2000$ (Invitrogen) according to the manufacturer's protocol [19]. Transfected cells were cultivated for two days at $30^{\circ} \mathrm{C}$ and then were fixed in $3.7 \%$ formaldehyde in PBS for $20 \mathrm{~min}$. The chambers were removed and the slides were sealed with cover slips after adding a Vectashield mounting medium (Vector Laboratories) with DAPI for nucleus staining. All images were collected by an LSM 510 confocal laser-scanning microscope (Zeiss) and processed using the Adobe Photoshop software.

\section{Evaluation of specific antibody productivity $\left(q_{\mathrm{Ab}}\right)$}

The specific antibody productivity $\left(q_{\mathrm{Ab}}\right)$ was evaluated as described previously [20]. The $q_{\mathrm{Ab}}$ was calculated from the plot of antibody concentration versus the time integral of viable cells during day 2 and day 4 .

\section{Competing interests}

The authors declare that they have no competing interests. 


\section{Authors' contributions}

YGK, JKJ, HWL, and EGL designed the research. YGK, BP, and JOA performed all the experiments and analyzed the data. YGK prepared the initial draft of the manuscript. EGL conceived of the study, coordinated all the components of the project, and prepared the final manuscript. All authors have read and approved the final manuscript.

\section{Acknowledgements}

This research was supported by the Converging Research Center Program through the National Research Foundation of Korea (NRF) funded by the Ministry of Education, Science and Technology (grant number: 20090082336).

Received: 31 January 2012 Accepted: 30 April 2012

Published: 15 May 2012

\section{References}

1. Browne SM, Al-Rubeai M: Selection methods for high-producing mammalian cell lines. Trends Biotechnol 2007, 25:425-432.

2. Brezinsky SC, Chiang GG, Szilvasi A, Mohan S, Shapiro RI, MacLean A, Sisk W, Thill G: A simple method for enriching populations of transfected $\mathrm{CHO}$ cells for cells of higher specific productivity. J Immunol Methods 2003, 277:141-155.

3. Holmes P, Al-Rubeai M: Improved cell line development by a high throughput affinity capture surface display technique to select for high secretors. J Immunol Methods 1999, 230:141-147.

4. Weaver JC, McGrath P, Adams S: Gel microdrop technology for rapid isolation of rare and high producer cells. Nat Med 1997, 3:583-585.

5. DeMaria CT, Cairns V, Schwarz C, Zhang J, Guerin M, Zuena E, Estes S, Karey KP: Accelerated clone selection for recombinant CHO CELLS using a FACS-based high-throughput screen. Biotechnol Prog 2007, 23:465-472.

6. Meng YG, Liang J, Wong WL, Chisholm V: Green fluorescent protein as a second selectable marker for selection of high producing clones from transfected CHO cells. Gene 2000, 242:201-207.

7. Yoshikawa T, Nakanishi F, Ogura Y, Oi D, Omasa T, Katakura Y, Kishimoto M, Suga Kl: Flow cytometry: an improved method for the selection of highly productive gene-amplified $\mathrm{CHO}$ cells using flow cytometry. Biotechnol Bioeng 2001, 74:435-442

8. Sleiman RJ, Gray PP, McCall MN, Codamo J, Sunstrom NA: Accelerated cell line development using two-color fluorescence activated cell sorting to select highly expressing antibody-producing clones. Biotechnol Bioeng 2008, 99:578-587.

9. Bianchi AA, McGrew JT: High-level expression of full-length antibodies using trans-complementing expression vectors. Biotechnol Bioeng 2003, 84:439-444.

10. Chen N, Ye Y, Zou J, Li S, Wang S, Martin A, Wohlhueter R, Yang JJ: Fluorescence complementation via EF-hand interactions. J Biotechnol 2009, 142:205-213.

11. Ghosh I, Hamilton AD, Regan L: Antiparallel leucine zipper-directed protein reassembly: application to the green fluorescent protein. $J \mathrm{Am}$ Chem Soc 2000, 122:5658-5659.

12. Lindman S, Johansson I, Thulin E, Linse S: Green fluorescence induced by EF-hand assembly in a split GFP system. Protein Sci 2009, 18:1221-1229.

13. Bochkov YA, Palmenberg AC: Translational efficiency of EMCV IRES in bicistronic vectors is dependent upon IRES sequence and gene location. Biotechniques 2006, 41:283-284.

14. Crameri A, Whitehorn EA, Tate E, Stemmer WP: Improved green fluorescent protein by molecular evolution using DNA shuffling. Nat Biotechnol 1996, 14:315-319.

15. Roederer M: Spectral compensation for flow cytometry: visualization artifacts, limitations, and caveats. Cytometry 2001, 45:194-205.

16. Kim SJ, Kim NS, Ryu CJ, Hong HJ, Lee GM: Characterization of chimeric antibody producing $\mathrm{CHO}$ cells in the course of dihydrofolate reductasemediated gene amplification and their stability in the absence of selective pressure. Biotechnol Bioeng 1998, 58:73-84.

17. Wilson CG, Magliery TJ, Regan L: Detecting protein-protein interactions with GFP-fragment reassembly. Nat Methods 2004, 1:255-262

18. Kim MS, Kim WH, Lee GM: A simple analysis system for the estimation of recombination efficiency using fluorescence-activated cell sorting. J Biotechnol 2007, 127:373-384.
19. Kim YG, Kim JY, Mohan C, Lee GM: Effect of Bcl-xL overexpression on apoptosis and autophagy in recombinant Chinese hamster ovary cells under nutrient-deprived condition. Biotechnol Bioeng 2009, 103:757-766.

20. Renard JM, Spagnoli R, Mazier C, Salles MF, Mandine E: Evidence that monoclonal antibody production kinetics is related to the integral of the viable cells curve in batch systems. Biotechnol Lett 1998, 10:91-96.

doi:10.1186/1472-6750-12-24

Cite this article as: Kim et al:: New cell line development for antibodyproducing Chinese hamster ovary cells using split green fluorescent protein. BMC Biotechnology 2012 12:24.

\section{Submit your next manuscript to BioMed Central and take full advantage of:}

- Convenient online submission

- Thorough peer review

- No space constraints or color figure charges

- Immediate publication on acceptance

- Inclusion in PubMed, CAS, Scopus and Google Scholar

- Research which is freely available for redistribution 